\title{
MRS study of meningeal hemangiopericytoma and edema: A comparison with meningothelial meningioma
}

\author{
VALERIA RIGHI $^{1,2}$, VITALIANO TUGNOLI ${ }^{1}$, ADELE MUCCI $^{2}$, ANTONELLA BACCI $^{3}$, \\ SERGIO BONORA $^{1}$ and LUISA SCHENETTI ${ }^{2}$ \\ ${ }^{1}$ Department of Biochemistry 'G. Moruzzi', University of Bologna, I-40126 Bologna; ${ }^{2}$ Department of Chemistry, \\ University of Modena and Reggio Emilia, I-41125 Modena; ${ }^{3}$ Division of Neuroradiology, \\ Department of Neuroscience, University of Bologna, I-40139 Bologna, Italy
}

Received February 28, 2012; Accepted May 3, 2012

DOI: 10.3892/or.2012.1919

\begin{abstract}
Intracranial hemangiopericytomas (HPCs) are rare tumors and their radiological appearance resembles that of meningiomas, especially meningothelial meningiomas. To increase the knowledge on the biochemical composition of this type of tumor for better diagnosis and prognosis, we performed a molecular study using ex vivo high resolution magic angle spinning (HR-MAS) magnetic resonance spectroscopy (MRS) perfomed on HPC and peritumoral edematous tissues. Moreover, to help in the discrimination between HPC and meningothelial meningioma we compared the ex vivo HR-MAS spectra of samples from one patient with HPC and 5 patients affected by meningothelial meningioma. Magnetic resonance imaging (MRI), in vivo localized single voxel ${ }^{1} \mathrm{H}$-MRS was also performed on the same patients prior to surgery and the in vivo and ex vivo MRS spectra were compared. We observed the presence of OH-butyrate, together with glucose in HPC and a low amount of $\mathrm{N}$-acetylaspartate in the edema, that may
\end{abstract}

Correspondence to: Dr Valeria Righi or Dr Vitaliano Tugnoli, Department of Biochemistry 'G. Moruzzi', University of Bologna, Via Belmeloro 8/2, I-40126 Bologna, Italy

E-mail: valeria.righi@unimore.it

E-mail: vitaliano.tugnoli@unibo.it

Abbreviations: Ala, alanine; Ac, acetate; CPMG, Carr-PurcellMeiboom-Gill; ChoCC, choline containing compounds; $\mathrm{Cr}$, creatine; HPCs, hemangiopericytomas; HR-MAS, high resolution magic angle spinning; MRS, magnetic resonance spectroscopy; MRI, magnetic resonance imaging; 1D, one-dimensional; 2D, two-dimensional; COSY, correlation spectroscopy; TOCSY, total correlation spectroscopy; HSQC, heteronuclear single quantum coherence; OH-But, $\beta$-hydroxybutyrate; Lac, lactate; Glx, glutamine plus glutamate; Glu, glutamate; Gln, glutamine; GSH, glutathione; Tau, taurine; Man, mannitol; Myo, myo-inositol; NAA, $N$-acetylaspartate; $\alpha$ - and $\beta$-Glc, $\alpha$ - and $\beta$-glucose; MM, macromolecules; HTau, hypotaurine; PC, phosphocholine

Key words: in vivo MRS, ex vivo HR-MAS MRS, brain, hemangiopericytoma, meningioma, edema, metabolic alteration reflect neuronal alteration responsible for associated epilepsy. Many differences between HPC and meningothelial meningioma were identified. The relative ratios of myo-inositol, glucose and gluthatione with respect to glutamate are higher in HPC compared to meningioma; whereas the relative ratios of creatine, glutamine, alanine, glycine and choline-containing compounds with respect to glutamate are lower in HPC compared to meningioma. These data will be useful to improve the interpretation of in vivo MRS spectra resulting in a more accurate diagnosis of these rare tumors.

\section{Introduction}

Hemangiopericytomas (HPCs) are rare, highly cellular and vascular tumors derived from pericytes of Zimmerman, which are contractile smooth-muscle cells surrounding the endothelium of the capillaries, and are ubiquitous in all types of mesenchymal tissue. HPCs may arise in the soft tissues anywhere in the body $(1,2)$ and the most common sites are head and neck, lower extremities and retroperitoneum. HPCs affecting the central nervous system are considered malignant $(3,4)$, existing in low-grade and high-grade form with predictably aggressive clinical behavior, including high rates of recurrence and distant metastases (5). Macroscopically, HPC is a solid tumor that is well demarcated from the adjacent brain tissue $(3,6)$. The tumor is typically treated by complete microsurgical removal, followed by local irradiation of the tumor bed. The majority of tumors can be removed in a seemingly complete manner. However, local recurrences are almost inevitable in the long run and tumor metastasis eventually occurs in over $60 \%$ of patients after 15 years $(3,6,7)$. Intracranial HPCs, classified under the heading of non-meningothelial mesenchymal tumors (8), are difficult to distinguish from meningiomas based on location, clinical presentation (9-11) and brain imaging $(5,12-14)$. Due to their more aggressive behavior, intracranial HPCs require different management and a correct diagnosis is critical (15-17). However, data from two studies $(18,19)$ suggest that the discrimination between HPC and meningiomas seems to be possible by using in vivo MR spectroscopy (MRS) and the authors hypothesized that the discrimination can be obtained based on the much higher levels of myo-inositol (Myo) in the HPCs. Moderately 
increased concentrations of Myo in the in vivo MR spectra of four HPCs have been also reported by Fountas et al (10).

Additional studies have reported on the in vivo ${ }^{1} \mathrm{H}-\mathrm{MRS}$ spectra from HPC, but they are not as informative as high resolution magic angle spinning (HR-MAS) MRS about the complete metabolic profile of this type of tumor. The choline containing compounds (ChoCC) peak is dominant in these in vivo spectra $(20,21)$.

HPCs are rare tumors, therefore performing a significant study on a large amount of samples in order to improve the diagnosis remains a challenge, however it is important to know more about the biochemical composition of this tumor. With this aim, we studied the metabolic profile of HPC using ex vivo HR-MAS MRS, which allows the whole analysis of intact tissue with minimal sample preparation (22-24). A comparison between the in vivo and ex vivo MRS spectra was performed with the goal to better understand HPC and edema tissues. Moreover, the difference between the HPC and meningothelial meningioma will be highlighted.

\section{Materials and methods}

Sample. Informed consent before spectroscopic examination was obtained from the patient. MRI and in vivo localized single voxel ${ }^{1} \mathrm{H}-\mathrm{MRS}$ were performed with a 3 Tesla wholebody scanner (General Electric Medical Systems, Milwaukee, WI) following the routine standard clinical protocol previously described (25). MR imaging was performed with T2-weighted fast spin-echo (FSE) sequences (TR, $4200 \mathrm{~ms}$; TE, $93 \mathrm{~ms}$; NEX, 2; 24-cm field of view; 512x512 matrix; 4-mm sections), fluid-attenuated inversion recovery (FLAIR) sequences (TR, $9002 \mathrm{~ms}$; TE, $91 \mathrm{~ms}$; NEX, 2; 24-cm field of view; 320x320 matrix; 4-mm sections) in the axial plane, and T1-weighted spin-echo (SE) sequences (TR, $560 \mathrm{~ms}$; TE, $18 \mathrm{~ms}$; NEX, 2; 24-cm field of view; $384 \times 224$ matrix; 4-mm sections) in the sagittal and coronal planes before administration of a contrast agent and coronal and axial planes after administration of a contrast agent. For choosing a voxel for spectroscopy, homogeneous regions on the T2-weighted image were selected carefully excluding cystic, necrotic, or hemorrhagic region. The volume of interest (VOI) size ranged from 1.2 to $8 \mathrm{~cm}^{3}$. ${ }^{1} \mathrm{H}-\mathrm{MR}$ spectra were acquired before administration of the contrast agent with a point-resolved spectroscopy sequence (PRESS) for localization, with TR $2000 \mathrm{~ms}$ and TE $35 \mathrm{~ms}, 128$ acquisitions, and a 3-pulse chemical shift selection suppression (CHESS) sequence to provide water suppression. We used automated optimization of gradient shimming, transmitter pulse power, and water suppression.

MRI revealed a left frontobasal perisellar lesion. The lesion is an extra-axial round mass with homogeneous intense enhancement after contrast administration and it is associated with severe cerebral edema in underlying brain parenchyma and midline shifts. According to Chernov et al (26) the extension of perilesional edema, evaluated by T2-weighted MR image, was graded as severe. The patient received mannitol as the only anti-edema drug. After MRI and in vivo MRS the patient underwent surgery and one sample was obtained from the lesion and a second sample was obtained from the edema area. A small portion of tumor and edema, were used for HR-MAS MRS analysis. A portion of tumoral specimen was used for routine histopathology and revealed a grade II WHO HPC (27). During surgery, resected tissue was sent for histological analysis, and remainder of the tissue was immediately frozen in liquid nitrogen and stored at $-80^{\circ} \mathrm{C}$ until MRS analyses. The manipulation time for sample storing under nitrogen is around $1 \mathrm{~min}$.

Ex vivo HR-MAS MRS. Before MRS examination, each sample was flushed with $\mathrm{D}_{2} \mathrm{O}$ to improve the homogeneity, the water suppression, and to add deuterium as a nucleus for the lock system. The sample was introduced in a MAS zirconia rotor (4 mm OD) maintained on ice, fitted with a 50- $\mu 1$ cylindrical insert to increase sample homogeneity, and then transferred into the probe cooled to $4^{\circ} \mathrm{C}$. Total time for sample preparation prior to NMR analysis was only a few minutes.

${ }^{1} \mathrm{H}$ and ${ }^{13} \mathrm{C}$ HR-MAS spectra were recorded with a Bruker Avance 400 (Bruker BioSpin, Karlsruhe, Germany) spectrometer operating at a frequency of 400.13 and $100.61 \mathrm{MHz}$, respectively. The instrument was equipped with a ${ }^{1} \mathrm{H},{ }^{13} \mathrm{C}$ HR-MAS probe. Experiments were performed at a temperature of $4^{\circ} \mathrm{C}$ controlled by a Bruker cooling unit.

Samples were spun at $4000 \mathrm{~Hz}$ and three different types of one-dimensional (1D) proton spectra were acquired by using the sequences implemented in the Bruker software: i) a composite pulse sequence (zgcppr) (28) with $1.5 \mathrm{~s}$ water presaturation during the relaxation delay, $8 \mathrm{kHz}$ spectral width, $32 \mathrm{k}$ data points, 32 scans, ii) a water-suppressed spinecho Carr-Purcell-Meiboom-Gill (CPMG) sequence (cpmgpr) (29) with $1.5 \mathrm{~s}$ water presaturation during the relaxation delay, $1 \mathrm{~ms}$ echo time $(\tau)$ and $360 \mathrm{~ms}$ total spin-spin relaxation delay

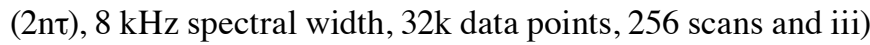
a sequence for diffusion measurements based on stimulated echo and bipolar-gradient pulses (ledbpgp2s1d) (30) with $\Delta 200 \mathrm{~ms}$, eddy current delay Te $5 \mathrm{~ms}, \delta 2 \times 2 \mathrm{~ms}$, sine-shaped gradient with $32 \mathrm{G} / \mathrm{cm}$ followed by a $200-\mu$ s delay for gradient recovery, $8 \mathrm{kHz}$ spectral width, 8k data points, 256 scans. Two-dimensional (2D) ${ }^{1} \mathrm{H},{ }^{1} \mathrm{H}$-correlation spectroscopy (COSY) $(31,32)$ spectra were acquired using a standard pulse sequence (cosygpprqf) and $0.5 \mathrm{~s}$ water presaturation during relaxation delay, $8 \mathrm{kHz}$ spectral width, $4 \mathrm{k}$ data points, 32 scans per increment, 256 increments. 2D ${ }^{1} \mathrm{H},{ }^{1} \mathrm{H}$-total correlation spectroscopy (TOCSY) $(33,34)$ spectra were acquired using a standard pulse sequence (mlevphpr) and $1 \mathrm{~s}$ water presaturation during relaxation delay, $100 \mathrm{~ms}$ mixing time (spin-lock), $4 \mathrm{kHz}$ spectral width, 4k data points, 32 scans per increment, 128 increments. 2D ${ }^{1} \mathrm{H},{ }^{13} \mathrm{C}$-heteronuclear single quantum coherence (HSQC) (35) spectra were acquired using an echoanti-echo phase-sensitive standard pulse sequence (hsqcetgp) and $0.5 \mathrm{~s}$ relaxation delay, $1.725 \mathrm{~ms}$ evolution time, $4 \mathrm{kHz}$ spectral width in $\mathrm{f} 2$, $4 \mathrm{k}$ data points, 128 scans per increment, $17 \mathrm{kHz}$ spectral width in $\mathrm{f} 1,256$ increments.

Relative quantification of metabolites. In each spectrum, relative ratios of all of the quantificable metabolites were calculated by measuring the area of individual metabolites signal with respect to the area of Glu signal at $2.34 \mathrm{ppm}$. The intensity of the Glu remained consistent in HPC and meningioma spectra as noted by the visual inspection of the ${ }^{1} \mathrm{H}$ spectra of all tissue samples. Metabolite ratios relative to Glu are expressed as mean $\pm \mathrm{SD}$ (standard deviation). 

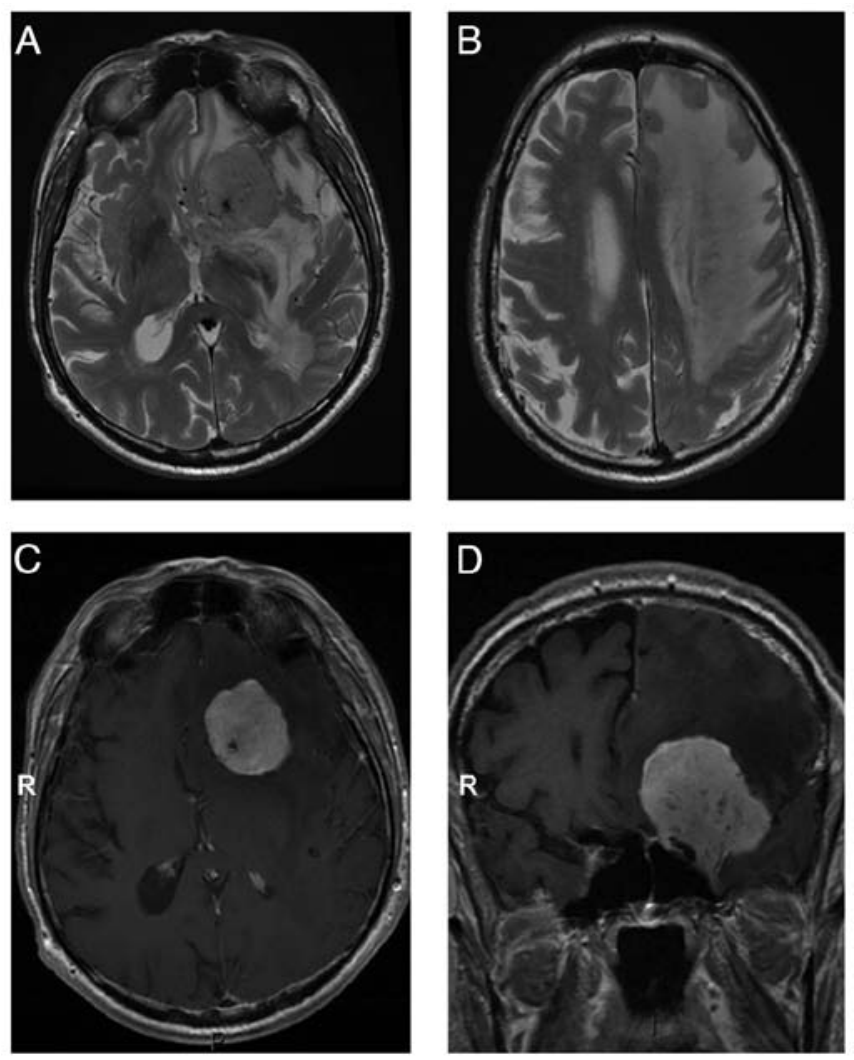

Figure 1. Axial T2-weighted MR images of HPC (A) and edema (B). Axial T1-weighted (C) and coronal T1-weighted (D) after contrast administration.

\section{Results}

Typical axial T2-weighted MR images of HPC, edema, axial T1-weighted and coronal T1-weighted MR images after contrast administration are shown in Fig. 1. The HPC demonstrate a large abnormal mass with severe edema. T1- and T2-weighted MR imaging characteristics, and contrast uptake appear compatible with both meningiomas and HPCs. The tumor demonstrates heterogeneous intensity being both hypoand isointense to white matter and iso- and hyperintense to gray matter. Small vascular elements are evident in the lesion (Fig. 1A). Significant edema in underlying brain parenchyma is present (Fig. 1B). The lesion shows intense contrast enhancement (Fig. 1C) and dural attachment (Fig. 1D). MRI shows a sharply demarcated extra-assial tumor with dural attachment in left para/suprasellar region and contrast enhancement on T1-weighted images after gadolinium. The lesion is isointense with grey matter on T2-weighted images with small hypointense areas due to vessels. Significant edema in underlying brain parenchyma is present.

Fig. 2 shows the $e x$ vivo $1 \mathrm{D}{ }^{1} \mathrm{H}$ HR-MAS MR spectra of HPC lesion. The spectrum (Fig. 2A) was obtained using a conventional ${ }^{1} \mathrm{H}$ spectrum with water presaturation, and it represents the global biochemical composition of lesion. The second spectrum (Fig. 2B), acquired with a spin-echo sequence, displays signals deriving from small metabolites. After the analysis several metabolites are identified: 1,2-propanediol (1,2-propanendiol is an exogenous compound used as solvent in pharmaceutical preparations containing some water-soluble ingredients), $\beta$-hydroxybutyrate (OH-But), lactate (Lac), alanine
A
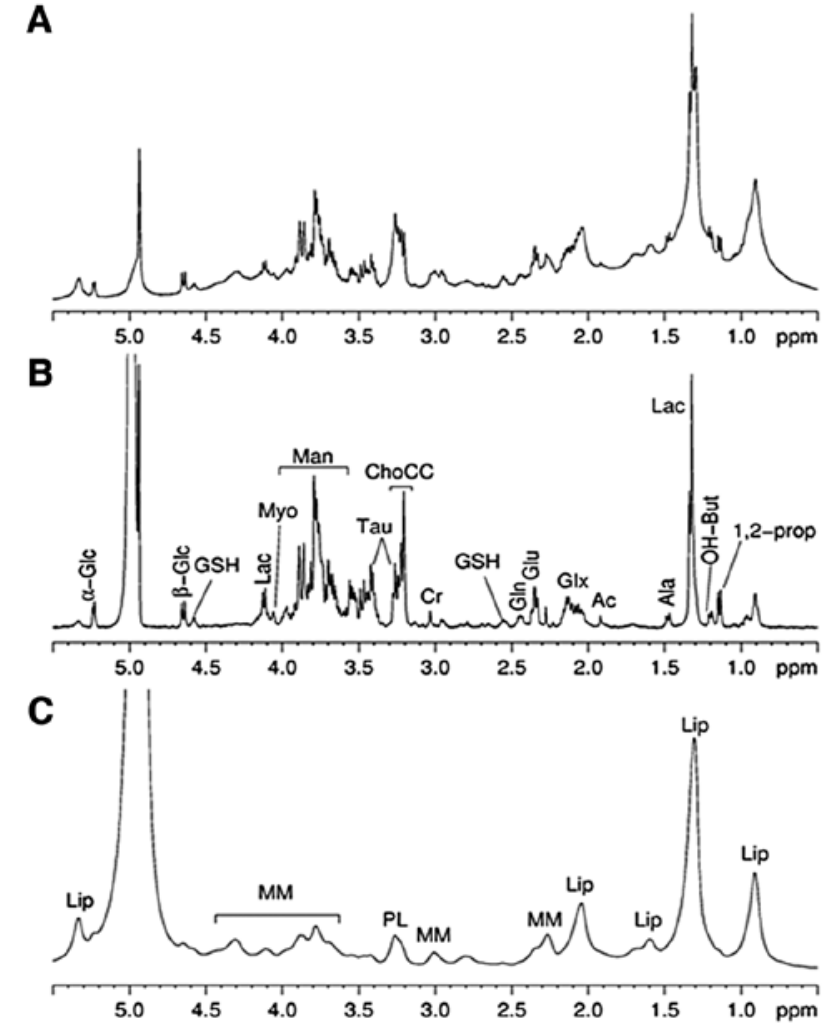

Figure 2. Ex vivo HR-MAS spectra of $\mathrm{HPC}$ lesion: (A) conventional ${ }^{1} \mathrm{H}$ spectrum with water presaturation; (B) ${ }^{1} \mathrm{H}$ CPMG spectrum; (C) ${ }^{1} \mathrm{H}$ diffusion edited spectrum.

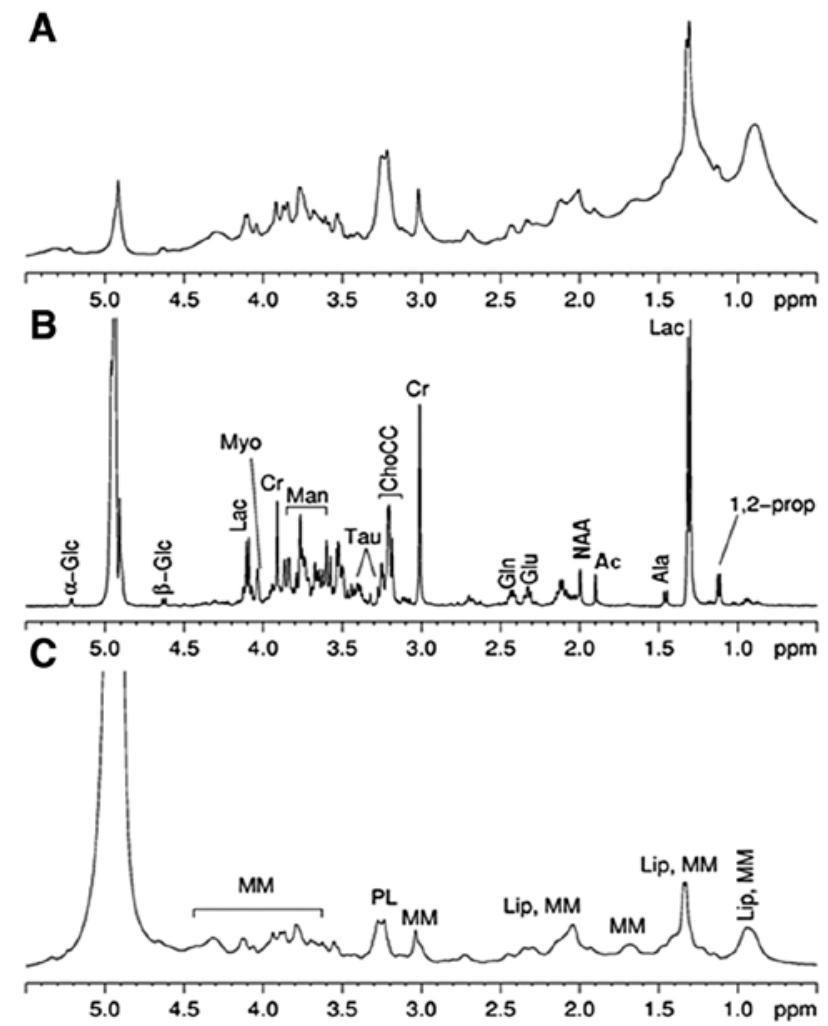

Figure 3. Ex vivo HR-MAS spectra of edema tissues around lesion: (A) conventional ${ }^{1} \mathrm{H}$ spectrum with water presaturation; (B) ${ }^{1} \mathrm{H}$ CPMG spectrum; (C) ${ }^{1} \mathrm{H}$ diffusion edited spectrum. 


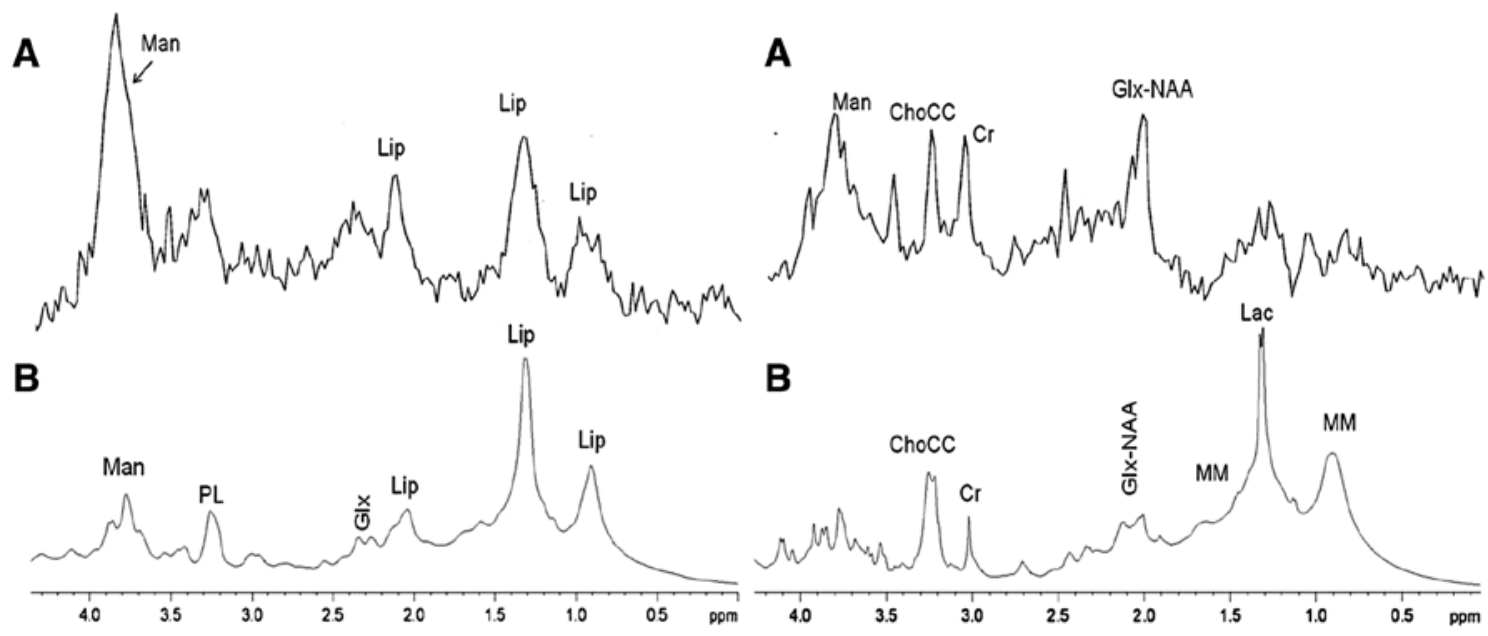

Figure 4. 1, The in vivo MRS (A) and ex vivo HR-MAS after line broadening (LB, 10) (B) spectra from HPC. 2, The in vivo MRS (A) and ex vivo HR-MAS after line broadening $(\mathrm{LB}, 5)(\mathrm{B})$ spectra from the edema.

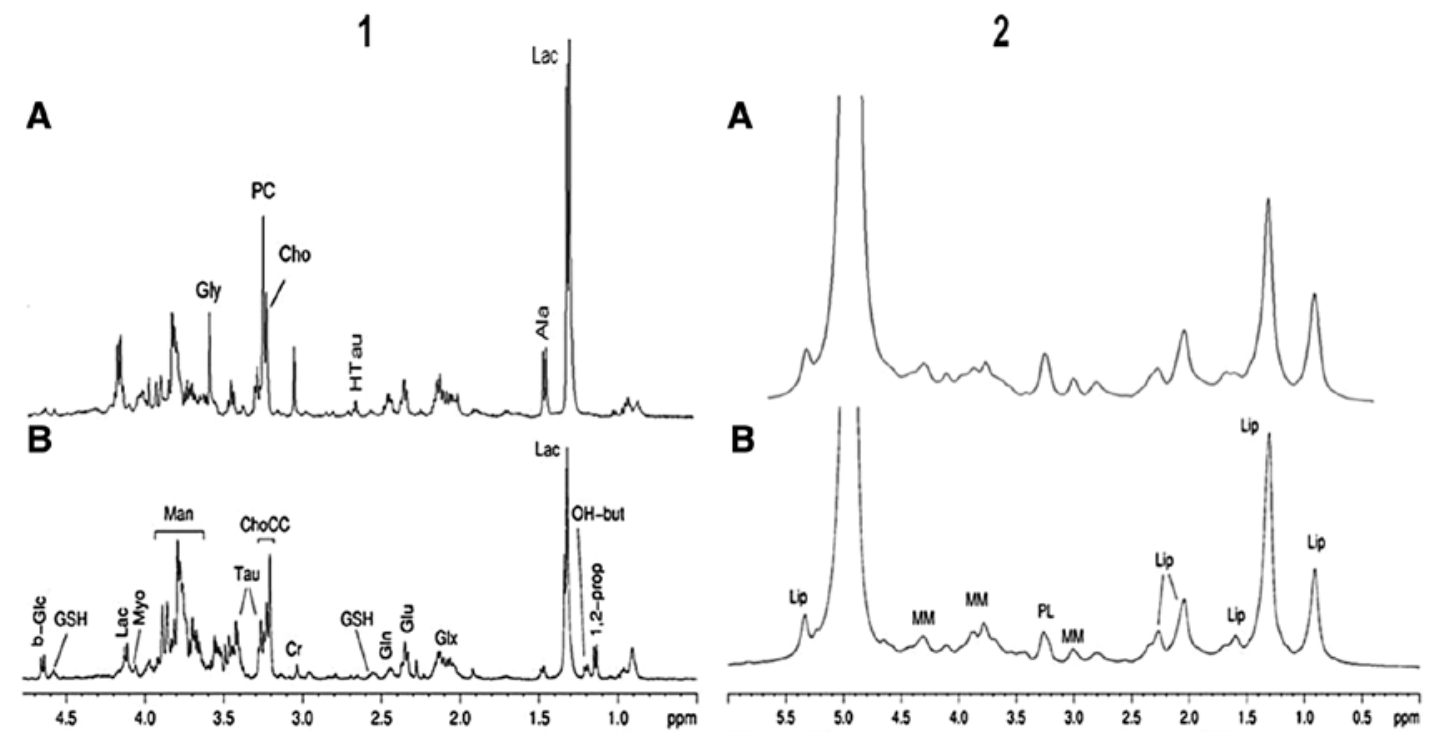

Figure 5. 1, Ex vivo HR-MAS ${ }^{1} \mathrm{H}$ CPMG spectra: comparison between meningioma (A) and HPC (B). 2, Ex vivo HR-MAS diffusion edited spectra: comparison between HPC (A) and meningioma (B).

(Ala), acetate (Ac), glutamine plus glutamate (Glx), glutamate (Glu), glutamine (Gln), glutathione (GSH), creatine $(\mathrm{Cr})$, ChoCC, taurine (Tau), mannitol (Man), Myo, $\alpha$ - and $\beta$-glucose ( $\alpha$ - and $\beta$-Glc). The assignment of 1,2-propanediol is based on the analysis of $1 \mathrm{D}{ }^{1} \mathrm{H}$, and selected $2 \mathrm{D}$ (COSY, TOCSY and HSQC) NMR spectra, where we highlight the H-H correlations of 1,2-propanediol. The signals are at $1.14 \mathrm{ppm}$ (d) $\mathrm{CH}_{3}$, 3.55/3.44 ppm $\mathrm{CH}_{2}$ and $3.87 \mathrm{ppm} \mathrm{CH}$. The spectrum (Fig. 2C) was acquired with a diffusion edited sequence and shows the contribution from lipids and macromolecules. The complete metabolic assignments were confirmed using two dimensional COSY, TOCSY and HSQC spectra. All of the experiments permit clarification of the metabolite pattern of the lesion and the same experiments were also used to characterize the metabolic profile of edema (Fig. 3).
The results were compared with data from the literature (36) and, when possible, with the experimental spectra in the Human Metabolome Database (37) to assign the different metabolites.

The same small metabolites were found in both tumor and edema, but in different amounts. For instance, the amount of $\mathrm{Cr}$ and Myo is lower in the lesion compared to in the edema, whereas levels of $\alpha$ - and $\beta$-Glc together with Tau seem to be higher in the lesion. Furthermore, we observed a different Glu/ Gln ratio in the two types of tissues, and that $\mathrm{OH}-\mathrm{But}$ and GSH were present only in HPC. Different profiles were observed for macromolecules (MM) and lipids: HPC diffusion spectrum (Fig. 2C) is characterized by the presence of lipids (manly triglycerides, probably as lipid bodies) and MM signals, whereas that of the edema (Fig. 3C) shows signals mainly due to MM. 
A direct comparison between in vivo (TE, 35) and conventional ex vivo spectra of the lesion after a suitable broadening process (Fig. 4, panel 1) shows that there is good agreement between the two profiles. The main difference between in vivo and ex vivo spectra is found in the Man signals due to the different magnetic fields used. At 3T, the field for in vivo MRS, the signals due to Man appear as a broad signal centred at $3.8 \mathrm{ppm}$, whereas at 9.4T, the field for ex vivo MRS spectra, Man signals are distributed in a region between 3.6 and $4.0 \mathrm{ppm}$ and structured at low line broadening. Man is an exogenous metabolite used to reduce brain pressure produced by the tumoral mass in patients.

The ex vivo HR-MAS spectrum of the edema (Fig. 4, panel 2B) shows more Lac and $\mathrm{MM}$ and less $\mathrm{N}$-acetylacetylaspartate (NAA) than the in vivo spectrum (Fig. 4, panel 2A). The Lac signal is higher in the ex vivo than in the in vivo spectrum of the edema, but this is likely not significant, since the Lac signal is always observed in ex vivo spectra due to the unavoidable period of ischemia during the biopsy procedure (38). The signal usually assigned to NAA in the in vivo spectrum at $2.01 \mathrm{ppm}$ in this case receives the contributions of Glu and Gln as can be seen from the HR-MAS ex vivo spectrum. The decrease in NAA and the increase in acetate (Ac) in the ${ }^{1} \mathrm{H}$ ex vivo HR-MAS spectrum of the edema are notable (Fig. 3B), suggesting the degradation of NAA to Ac and aspartate (39).

Comparing the HR-MAS spectra of HPC and meningioma (Fig. 5), we observe many differences between the two tumors. The phosphocholine (PC)/choline (Cho) ratio appears to be different comparing meningioma to HPC (Fig. 5, panel 1), but in our opinion this aspect needs further investigation. In fact, we observed a high variability of $\mathrm{PC} / \mathrm{Cho}$ in our meningioma set. It is to be noted that HR-MAS MRS allows the detection of Ala in HPC, which has been reported to be absent $(19,20)$. This is probably due to the higher sensitivity of HR-MAS MRS with respect to in vivo MRS. Nevertheless, both techniques show that the Ala content is lower in HPC than in meningioma. The diffusion edited spectra of HPC and meningioma are shown in Fig. 5, panel 2. The comparison shows a very similar profile with slightly higher amounts of lipids in HPC compared to meningioma.

\section{Discussion}

We applied ex vivo HR-MAS MRS to assess the metabolic status of HPC and edema. Several differences in the metabolic profile of HPC and edema can be detected.

The resonance deriving from the OH-But was identified in the lesion spectrum (Fig. 2B). It has been shown that elevated ketone bodies can target brain tumors while enhancing the metabolic efficiency of normal neurons glia. Moreover, the ketones bodies are toxic to some tumor cells and they might restrict availability of glutamine (40-42). In our case, the presence of $\mathrm{OH}-\mathrm{But}$ together with Glc in the lesion could suggest the bioenergetic transition from normal to neoplastic brain. In contrast to a normal brain, which oxidizes Glc as well as ketone bodies for energy, malignant brain tumors are largely dependent on Glc for energy. OH-But is also reported to prolong cell survival time and to inhibit cerebral edema by improving energy metabolism in the hypoxia, anoxia and global cerebral
Table I. Relative ratios metabolite/Glu from CPMG HR-MAS experiments in HPC and MN (meningothelial meningioma).

\begin{tabular}{lcc}
\hline Metabolites & $\begin{array}{c}\text { HPC } \\
\text { (1 sample) }\end{array}$ & $\begin{array}{c}\text { MN } \\
\text { (5 samples) }\end{array}$ \\
\hline Ala & 0.3 & $1.01 \pm 0.42$ \\
Gln & 0.4 & $0.77 \pm 0.34$ \\
Cr & 0.3 & $0.59 \pm 0.38$ \\
ChoCC & 1.84 & $3.39 \pm 1.51$ \\
Gly & 0.14 & $0.93 \pm 0.31$ \\
Myo & 0.15 & $0.09 \pm 0.08$ \\
Glc & 0.47 & - \\
GSH & 0.24 & - \\
\hline
\end{tabular}

$\mathrm{MN}$ values are expressed as mean $\pm \mathrm{SD}$ (standard deviation).

ischemia models. The cerebroprotective effect of OH-But was examined in rats with permanent (p)-occlusion and transient (t)-occlusion of middle cerebral artery (MCA) (43).

The low intensity of the NAA signal (singlet at $2.01 \mathrm{ppm}$; Fig. 3B) is evident in peritumoral edema tissue analyzed in ex vivo, in agreement with the decrease of NAA in peritumoral lesion reported in in vivo spectra MRS $(25,44)$. The NAA is belived to be a marker of neuronal integrity (45), and its decrease in the edema may reflect neuronal alteration responsible for associated epilepsy. Our HR-MAS data obtained from biopsy samples confirm a strong neuronal alteration and the patient showed neurological disorders, including seizure epilepsy. Regional brain reduction of NAA levels measured by MRS is a well recognized marker of neuronal or axonal loss in many neurologic disorders, including traumatic brain injury, ischemic stroke, epilepsy, multiple sclerosis, neoplastic and non-neoplastic lesions $(46,47)$.

Another important difference between HPC and edema is the $\mathrm{Cr}$ amount, which was very low in HPC and higher in the edema. This is in agreement with our previous in vivo MRS study of peritumoral brain edema where an energy-linked metabolic alteration was associated with injury to the myelin sheath (25).

The biochemical profile of HPC can be compared with that of meningioma, the molecular characterization of which, by ex vivo HR-MAS MRS, has already been reported $(48,49)$. The more suitable comparison is between HPC and meningothelial meningioma, which is the most common meningioma. We used the Glu signal at $2.34 \mathrm{ppm}$ as a reference for the analysis because this signal does not overlap with others and it is easy to integrate. Moreover, we did not observe appreciable differences among the meningothelial meningioma (grade I) and HPC samples.

We observed that the relative ratios of Myo, Glc and GSH with respect to Glu are higher in HPC compared to meningioma; and vice versa the relative ratios of $\mathrm{Cr}$, Gln, Ala, Gly and $\mathrm{ChoCC}$ with respect to Glu are lower in HPC compared to meningioma (Table I).

Hypotaurine (HTau) is detected only in meningioma, whereas OH-But is present only in HPC. Overall, these data 
are consistent with published studies (19-21). It is difficult to quantify the Tau content in HPC due to overlapping signal from $\alpha-, \beta-$ Glc.

In conclusion, HR-MAS spectra permit the characterization of the metabolic profiles of HPC and peritumoral edema tissue. The presence of $\mathrm{OH}$-But together with Glc in the lesion could suggest the bioenergetic transition from normal to neoplastic brain. The variations in the NAA amount in the edema area may reflect neuronal alteration responsible for associated epilepsy. Our HR-MAS data performed on biopsy confirmed a strong neuronal alteration, and the patient showed neurological disorders including seizure epilepsy.

We identified many differences between HPC and meningioma. The relative ratios of Myo, Glc, and GSH with respect to Glu are higher in HPC compared to meningioma; and, vice versa, the relative ratios of $\mathrm{Cr}$, Gln, Ala, Gly and ChoCC with respect to Glu are lower in HPC compared to meningioma. Ala was detected by HR-MAS MRS in HPC but not in vivo MRS.

The ex vivo HR-MAS allows for a comprehensive metabolic characterization of HPC tissues. We hope that identification of key metabolites would provide a basis for studies aimed at better understanding of the biochemical processes of this tumor. These data obtained from a large number of samples would be useful to improve the interpretation of in vivo MRS spectra and allow for better diagnosis and prognosis.

\section{Acknowledgements}

This study was supported by a grant of MUR ex $60 \%$ to V.T. and from a L'Oréal-Unesco Fellowship to Valeria Righi.

\section{References}

1. Espat NJ, Lewis JJ, Leung D, et al: Conventional hemangiopericytoma: modern analysis of outcome. Cancer 95: 1746-1751, 2002.

2. Koch M, Nielsen GP and Yoon SS: Malignant tumors of blood vessels: angiosarcomas, hemangioendotheliomas, and hemangiopericytomas. J Surg Oncol 97: 321-329, 2008.

3. Mena H, Ribas JL, Pezeshkpour GH, Cowan DN and Parisi JE: Hemangiopericytoma of the central nervous system: a review of 94 cases. Hum Pathol 22: 84-91, 1991.

4. Scheithauer BW, Fuller GN and VandenBerg SR: The 2007 WHO classification of tumors of the nervous system: controversies in surgical neuropathology. Brain Pathol 18: 307-316, 2007.

5. Wu W, Shi JX, Cheng HL, et al: Hemangiopericytomas in the central nervous system. J Clin Neurosci 16: 519-523, 2009.

6. Jääskeläinen J, Louis DN, Paulus W and Haltia MJ: Haemangiopericytoma. In: Pathology and Genetics of the Nervous System Tumors. Kleihues P and Cavenee WK (eds). WHO Classification of Tumors. IARC Press, Lyon, pp190-192, 2000.

7. Kim JH, Jung HW, Kim YS, et al: Meningeal hemangiopericytomas: long-term outcome and biological behavior. Surg Neurol 59: 47-54, 2003.

8. Kleihaus P, Louis DN, Burger PC, et al: The WHO classification of tumors of the nervous system. J Neuropathol Exp Neurol 61 215-229, 2002

9. Galanis E, Buckner JC, Scheithauer BW, et al: Management of recurrent meningeal hemangiopericytoma. Cancer 82: 1915-1920, 1998.

10. Fountas KN, Kapsalaki E, Kassam M, et al: Management of intracranial meningeal hemangiopericytomas: outcome and experience. Neurosurg Rev 29: 145-153, 2006.

11. Sundaram C, Uppin SG, Uppin MS, et al: A clinicopathological and immunohistochemical study of central nervous system hemangiopericytomas. J Clin Neurosci 17: 469-472, 2010.
12. Akiyama M, Sakai H, Onoue H, Miyazaki $Y$ and Abe T: Imaging intracranial hemangiopericytomas: study of seven cases. Neuroradiology 46: 194-197, 2004.

13. Sibtain NA, Butt $S$ and Connor SE: Imaging features of central nervous system hemangiopericytomas. Eur Radiol 17: 1685-1693, 2007.

14. Tashjian VS, Khanlou N, Vinters HV, et al: Hemangiopericytoma of the cerebello pontine angle: a case report and review of the literature. Surg Neurol 72: 290-295, 2009.

15. Soyuer S, Chang EL, Selek U, et al: Intracranial meningeal hemangiopericytoma: the role of radiotherapy: report of 29 cases and review of the literature. Cancer 100: 1491-1497, 2004.

16. Olson C, Yen CP, Schlesinger D and Sheehan J: Radiosurgery for intracranial hemangiopericytoma: outcomes after initial and repeat Gamma Knife surgery. J Neurosurgery 112: 133-139, 2010.

17. Rutkowski MJ, Sughrue ME, Kane AJ, et al: Predictors of mortality following treatment of intracranial hemangiopericytoma. Neurosurgery 113: 340-351, 2010.

18. Barba I, Moreno A, Martinez-Pérez I, et al: Magnetic resonance spectroscopy of brain hemangiopericytomas: high myoinositol concentrations and discrimination from meningiomas. $\mathrm{J}$ Neurosurg 94: 55-60, 2001.

19. Fortuniak J, Jaskólski DJ, Stefańczyk L, Zawirski M and Gajewicz W: Magnetic resonance imaging of rare intracranial neoplasms - role of the in vivo $1 \mathrm{~h}$ spectroscopy in the radiological differential diagnostics. Cen Eur Neurosurg 71: 181-188, 2010.

20. Cho YD, Choi GH, Lee SP and Kim JK: (1)H-MRS metabolic patterns for distinguishing between meningiomas and other brain tumors. Magn Reson Imaging 21: 663-672, 2003.

21. Hattingen E, Pilatus U, Good C, et al: An unusual intraventricular haemangiopericytoma: MRI and spectroscopy. Neuroradiology 45: 386-389, 2003

22. Beckonert O, Coen M, Keun HC, et al: High-resolution magic-angle-spinning NMR spectroscopy for metabolic profiling of intact tissue. Nat Protoc 5: 1019-1032, 2010.

23. DeFeo EM and Cheng LL: Charactrizing human cancer metabolomics by ex vivo 1HRMAS MRS. Technol Cancer Res Treat 9: 381-391, 2010.

24. Moestue S, Sitter B, Bathen TF, Tessem MB and Gribbestad IS: HR MAS MR spectroscopy in metabolic characterization of cancer. Curr Top Med Chem 11: 2-26, 2011.

25. Ricci R, Bacci A and Tugnoli V, et al: Metabolic findings on $3 \mathrm{~T}{ }^{1} \mathrm{H}-\mathrm{MR}$ spectroscopy in peritumoral brain edema. Am J Neuroradiol 28: 1287-1291, 2007.

26. Chernov MF, Kubo O, Hayashi M, et al: Proton MRS of the peritumoral brain. J Neurol Sci 228: 137-142, 2005.

27. Louis DN, Ohgaki H, Wiestler OD, et al: The 2007 WHO classification of tumours of the central nervous system. Acta Neuropathol 114: 97-109, 2007.

28. Bax A: A spatially selective composite $90^{\circ}$ radiofrequency pulse. J Magn Reson 65: 142-145, 1985.

29. Meiboom S and Gill D: Modified spin-echo method for measuring nuclear relaxation time. Rev Sci Instrum 20: 688-691, 1958.

30. Wu D, Chen A and Johnson CS Jr: An improved diffusion ordered spectroscopy experiment incorporating bipolar gradient pulses. Magn Reson Series A 115: 260-264, 1995.

31. Jeener J: Pulse pair techniques in high resolution NMR. In: Ampere International Summer School. Basko Polje, 1971.

32. Aue WP, Bartholdi E and Ernst RR: Two-dimensional spectroscopy. Application to nuclear magnetic resonance. J Chem Phys 64: 2229-2246, 1976.

33. Braunschweiler L and Ernst RR: Coherence transfer by isotropic mixing: application to proton correlation spectroscopy. J Magn Reson 53: 521-528, 1983.

34. Bax A and Davis DG: MLEV-17-based two-dimensional homonuclear magnetization transfer spectroscopy. J Magn Res 65: 355-360, 1985.

35. Bodenhausen G and Ruben DJ: Natural abundance nitrogen- 15 NMR by enhanced eteronuclear spectroscopy. Chem Phys Lett 69: 185-189, 1980.

36. Fan TMW: Metabolite profiling by one- and two-dimensional NMR analysis of complex mixtures. Prog Nuc Mag Res Spec 28: 161-219, 1996.

37. Wishart DS, Tzur D, Knox C, et al: HMDB: the human metabolome database. Nucleic Acids Res 35: D521-D526, 2007.

38. Opstad KS, Wright AJ, Bell BA, et al: Correlations between in vivo ${ }^{1} \mathrm{H}$ MRS and ex vivo ${ }^{1} \mathrm{H}$ HRMAS metabolite measurements in adult human gliomas. J Magn Reson Imag 31: 289-297, 2010. 
39. Harting I, Jost G, Hacke $\mathrm{N}$ and Hartmann M: Magnetic resonance spectroscopy of brain tumours. Nervenarzt 76: 403-417, 2005.

40. Kashiwaya Y, Pawlosky R, Markis W, et al: A ketone ester diet increases brain malonyl-CoA and uncoupling proteins 4 and 5 while decreasing food intake in the normal Wistar Rat. J Biol Chem 285: 25950-25956, 2010.

41. Yudkoff M, Daikhin Y, Melø TM, et al: The ketogenic diet and brain metabolism of amino acids: relationship to the anticonvulsant effect. Annul Rev Nutr 27: 415-430, 2007.

42. Skinner R, Trujillo A, Ma X and Beierle EA: Ketone bodies inhibit the viability of human neuroblastoma cells. J Pediatr Surg 44: 212-216, 2009.

43. Suzuki M, Suzuki M, Kitamura Y, et al: $\beta$-hydroxybutyrate, a cerebral function improving agent, protects rat brain against ischemic damage caused by permanent and transient focal cerebral ischemia. Jpn J Pharmacol 89: 36-43, 2002.

44. Chernov MF, Kawamata T, Amano K, et al: Possible role of single-voxel (1)H-MRS in differential diagnosis of suprasellar tumors. J Neurooncol 91: 191-198, 2009.
45. Demougeot C, Garnier P, Mossiat C, et al: $N$-Acetylaspartate, a marker of both cellular dysfunction and neuronal loss: its relevance to studies of acute brain injury. J Neurochem 77: 408-415, 2001.

46. Moffett JR, Ross B, Arun P, et al: $N$-Acetylaspartate in the CNS: from neurodiagnostics to neurobiology. Prog Neurobiol 81: 89-131, 2007.

47. Rigotti DJ, Inglese $\mathrm{M}$ and Gonen O: Whole-brain $N$-acetylaspartate as a surrogate marker of neuronal damage in diffuse neurologic disorders. Am J Neuroradiol 28: 1843-1849, 2007.

48. Tugnoli V, Schenetti L, Mucci A, et al: Ex vivo HR-MAS MRS of human meningiomas: a comparison with in vivo ${ }^{1} \mathrm{H}$ MR spectra. Int J Mol Med 18: 859-869, 2006.

49. Monleon D, Morales JM, Gonzalez-Darder J, et al: Benign and atypical meningioma metabolic signatures by high-resolution magic-angle spinning molecular profiling. J Proteome Res 7: 2882-2888, 2008. 